\title{
Hematopoietic stem cell-derived exosomes promote hematopoietic differentiation of mouse embryonic stem cells in vitro via inhibiting the miR126/Notch1 pathway
}

\author{
Feng-ling LIAO ${ }^{1, \#}$, Lin TAN ${ }^{1, \#}$, Hua LIU ${ }^{2}$, Jin-ju WANG ${ }^{4}$, Xiao-tang MA ${ }^{3}$, Bin $\mathrm{ZHAO}^{3}$, Yanfang $\mathrm{CHEN}^{3,4}, \mathrm{Ji} \mathrm{BIHL}^{4}$, Yi YANG ${ }^{2}$, Ri- \\ ling $\mathrm{CHEN}^{1, *}$ \\ ${ }^{1}$ Department of Pediatrics, the Affiliated Hospital of Guangdong Medical University, Zhanjiang 524001, China; ${ }^{2}$ College of Health \\ Science, Wuhan Sports University, Wuhan 430079, China; ${ }^{3}$ Institute of Neurology, the Affiliated Hospital of Guangdong Medical \\ University, Zhanjiang 524001, China; ${ }^{4}$ Department of Pharmacology \& Toxicology, Boonshoft School of Medicine, Wright State \\ University, Dayton, $\mathrm{OH} 45435$, USA
}

\begin{abstract}
Cell-derived exosomes (EXs) can modulate target cell differentiation via microRNAs (miRs) that they carried. Previous studies have shown that miR126 is highly expressed in hematopoietic stem cells (HSCs) and plays a role in hematopoiesis via modulating the Notch pathway that participates in progenitors' cell fate decisions. In this study we investigated whether HSC-derived EXs (HSCEXs) could affect the differentiation of mouse embryonic stem cells (ESCs) into HSCs. We prepared HSC-EXs ${ }^{\text {con }}$, HSC-EXs ${ }^{\text {sc }}$ and HSCEXs $^{\text {miR126 }}$ from control HSCs and the HSCs transfected with scramble control or miR126 mimics, respectively. HSC-EXs were isolated by ultracentrifugation and analyzed using nanoparticle tracking analysis. We incubated the collected EXs with mouse ESCs over a 10-d differentiation induction period, during which HSC-EXs and a Notch pathway activator (Jagged1, $100 \mathrm{ng} / \mathrm{mL}$ ) were added to the cultures every $3 \mathrm{~d}$. After the 10-d differentiation period, the expression levels of miR126, SSEA1, CD117, Sca1, Notch1 and Hes1 in ESCs were assessed. The generated HSCs were validated by flow cytometry using antibodies against HSC markers (CD117, CD34 and Sca1). Our results revealed that: (1) transfection with miR126 mimics significantly increased miR126 levels in HSC-EXs ${ }^{\text {miR126. }}$. (2) HSC-EX co-culture promoted mouse ESCs differentiation into HSCs with the most prominent effect found in the HSC-EXs ${ }^{\text {miR126 }}$ co-culture. (3) HSC differentiation was verified by reduced SSEA1 expression and increased CD117 and Sca1 expression. (4) All the effects caused by HSCEXs were accompanied by significant reduction of Notch1 and Hes1 expression, thus inhibition of the Notch1/Hes1 pathway, whereas activation of Notch by Jagged1 abolished the effects of HSC-EXs ${ }^{\text {miR126 }}$. In conclusion, HSC-EXs promote hematopoietic differentiation of mouse ESCs in vitro by inhibiting the miR126/Notch1 pathway.
\end{abstract}

Keywords: hematopoietic stem cells; embryonic stem cells; differentiation; exosomes; miR126; Notch; Jagged1

Acta Pharmacologica Sinica (2018) 39: 552-560; doi: 10.1038/aps.2017.130; published online 19 Oct 2017

\section{Introduction}

Hematopoietic disease affects millions of people worldwide and can be treated by transplantation of hematopoietic stem cells (HSCs). However, the difficulty in collecting a sufficient number of HSCs from bone marrow and the lack of an efficient in vitro approach for HSC expansion have impeded the clinical use of HSC-based therapy ${ }^{[1]}$. Embryonic stem cells

\footnotetext{
\# These authors contributed equally to this work.

* To whom correspondence should be addressed.

E-mail chenr1319@163.com

Received 2017-03-25 Accepted 2017-06-18
}

(ESCs) derived from the blastocyst of the early embryo could provide a source of HSCs ${ }^{[2]}$.

Exosomes (EXs) are extracellular vesicles secreted by various types of cells ${ }^{[3-5]}$. Accumulating studies have demonstrated that extracellular vesicles such as EXs are important intercellular mediators and can alter the functions of target cells by conveying information from their cargoes, such as microRNAs (miRs) ${ }^{[5-7]}$. For example, Xin et al reported that EXs from mesenchymal stem cells can promote neural plasticity by transferring miR133b to target cells in an in vivo stroke model ${ }^{[7]}$. The extracellular vesicles derived from endothelial progenitor cells can protect endothelial cells against hypoxia/reoxygenation 
injury by modulating miR126 expression ${ }^{[5]}$. Recently, our group found that endothelial progenitor cell-derived vesicles from healthy controls protected the functions of endothelial progenitor cells from diabetic patients via miR126 carried within the vesicles ${ }^{[8]}$. Interestingly, Notch signaling pathway proteins, such as the ligand Delta-like 4, can be packaged into endothelial cell-derived EXs and transferred to other endothelial cells to inhibit Notch signaling in the recipient cells. In addition, EXs can alter recipient cell differentiation. It has been shown that EXs derived from neuronal progenitor cells can promote neuronal differentiation from mesenchymal stem cells $^{[9]}$. Ismail et al reported that macrophage-derived extracellular vesicles promoted naïve differentiation into macrophage $\mathrm{e}^{[10]}$. However, whether HSC-derived EXs (HSC-EXs) can promote hematopoietic differentiation from ESCs remains unknown.

miRs are considered as master regulators of cellular processes, including cell differentiation. Previous studies have demonstrated that several miRs, such as miR142, miR181a, are predominately expressed in hematopoietic cells ${ }^{[11,12]}$. Among these miRs, miR126 is highly expressed and functional within the HSC compartments ${ }^{[13]}$. It has been shown that miR126 is progressively downregulated during the early stage of hematopoietic commitment ${ }^{[14]}$, indicating its role in HSC differentiation. Overexpression of miR126 in ESCs resulted in a reduction in the number of erythroid colonies, suggesting an inhibitory role of miR126 in erythropoiesis ${ }^{[11]}$. Nevertheless, only a few studies have investigated the role of miR126 in HSC differentiation by ESCs.

Recently, Huang et al demonstrated that overexpressing miR126 could enhance mesenchymal stem cells angiogenesis by upregulating the Notch ligand, Delta-like $4^{[15]}$, suggesting that miR126 could affect the Notch signaling pathway in stem cells. It has been well documented that Notch signal transduction is based ligand (D111, D113, D114, Jagged1, and Jagged2) binding to the appropriate receptor (Notch1 to 4$)^{[16]}$. The Notch signaling pathway governs fundamental processes such as cellular differentiation, proliferation and development ${ }^{[17]}$. For example, activation of the Notch pathway by Jagged 1 can promote ESCs differentiation into neural cells ${ }^{[18]}$. Previous studies have shown that Notch signaling plays an important role in progenitors' cell fate decisions ${ }^{[19,20]}$ and in the development of the hematopoietic system ${ }^{[21-23]}$. For example, Notch1 activation could result in the differentiation of bone marrow cells into T cells ${ }^{[19]}$, and Jagged1 (a Notch ligand) can enhance the differentiation of mesenchymal stem cells into cardiomyocytes $^{[20]}$. Activation of Notch1 signaling inhibits the differentiation of $\mathrm{Sca}^{+}$lin ${ }^{-}$bone marrow cells (a population of HSCs), thus impeding the cells from exiting the stem cell stage into the progenitor cell stage $\mathrm{e}^{[21]}$ and indicating that HSC expansion can be achieved via Notch pathway activation. Since less information is available regarding to the role of Notch in hematopoietic generation from ESCs, we investigated whether the Notch signaling pathway was involved in HSC-EXs effects on hematopoietic differentiation in ESCs.

In this study, we aimed to investigate whether the EXs from HSCs (HSC-EXs) can promote HSC differentiation from ESCs via modulation of the miR126/Notch pathway.

\section{Materials and methods Culture of ESCs}

Mouse ESCs were purchased from Cyagen Biosciences. The ESCs were cultured and maintained on feeder layers of mitomycin C-treated mouse embryonic fibroblasts (MEFs) in standard ESC complete medium containing knockout highglucose DMEM supplemented with $15 \%$ fetal bovine serum (FBS), 1\% nonessential amino acids, $1 \mathrm{mmol} / \mathrm{L}$ sodium pyruvate, $0.1 \mathrm{mmol} / \mathrm{L} 2$-mercaptoethanol, $2 \mathrm{mmol} / \mathrm{L}$ glutamine, and $1000 \mathrm{U} / \mathrm{mL}$ leukemia inhibitory factor (LIF). The culture medium was changed daily. ESCs were regularly passaged every 48-72 h at a proportion of 1:10 on mitomycin C-treated MEF layers after dissociation using Tryple Express (Life Technologies). ESCs were cultured to form embryonic bodies (EBs) for induction of HSC differentiation ${ }^{[24]}$. In brief, ESCs were dissociated and cultured with ESC complete medium without leukemia inhibitory factor (LIF) on sterile bacteriological grade petri dish for $4 \mathrm{~d}$. The resultant EBs were used for the differentiation study.

\section{Preparation and collection of EXs released from HSCs}

To overexpress miR126, HSCs (obtained from ATCC) were transfected with miR126 mimics or a miR126 scramble control (sc, $1 \mathrm{nmol} / \mathrm{L}$, Thermo Fisher Scientific) for $24 \mathrm{~h}$ using Lipofectamine 2000 according to the manufacturer's instructions ${ }^{[8]}$. After the $24 \mathrm{~h}$ transfection, the cell culture medium was collected for HSC-EXs isolation. In brief, cell culture medium was collected and centrifuged at $300 \times g$ for $15 \mathrm{~min}$, followed by $2000 \times g$ for $30 \mathrm{~min}$ to remove cells and cell debris. Then, the cell-free culture medium was centrifuged at $20000 \times g$ for $70 \mathrm{~min}$, followed by ultracentrifugation at $170000 \times \mathrm{g}$ for $6 \mathrm{~h}$ to pellet the EXs ${ }^{[25]}$. The pelleted HSC-EXs ${ }^{\text {con }}, \mathrm{HSC}_{-} \mathrm{EXs}{ }^{\mathrm{miR} 126}$, and HSC-EXs ${ }^{\text {sc }}$ were resuspended in phosphate-buffered saline (PBS) that had been passed through a $20 \mathrm{~nm}$-filtered (Whatman, Pittsburgh, PA, USA), aliquoted for nanoparticle tracking analysis (NTA) and co-culture experiments.

\section{Nanoparticle tracking analysis of EXs}

The NanoSight NS300 with a 405-nm laser instrument (Malvern Instruments, UK) was used to detect EXs as previously reported $^{[25]}$. Briefly, to determine particle size and concentration, the collected HSC-EXs were resuspended with $700 \mu \mathrm{L}$ of filtered PBS and analyzed using the NTA light-scatter mode. To detect CD63 expression in the EXs, the collected EXs were incubated with CD63-conjugated microbeads $(10 \mu \mathrm{L}$; Miltenyi Biotec) in a $100-\mu \mathrm{L}$ reaction volume for $2 \mathrm{~h}$. A magnet (DynaMag-2 magnet; Life Technologies) was then applied to separate the $\mathrm{CD}^{+}{ }^{+} \mathrm{EXs}$ from the total EX suspension. After an overnight magnet separation, the $\mathrm{CD}^{+} 3^{+} \mathrm{EXs}$ were resuspended in $100 \mu \mathrm{L}$ of filtered PBS and incubated with rabbit anti-goat IgG conjugated with Q-dot 655 (1:350; Life Technologies) for $90 \mathrm{~min}$ at RT. All samples were analyzed using the NTA fluorescence-scatter mode. Three videos of typically $30 \mathrm{~s}$ duration were taken with a frame rate of 30 frames per second. 
The Data were analyzed using NTA 3.0 software (Malvern Instruments) on a frame-by-frame basis.

\section{Differentiation of HSCs from ESCs}

The formed EBs were collected using a cut tip and trypsinized with TrypLE Express (Life Technologies). The EBs were then centrifuged and resuspended with HSC induction medium (knockout high-glucose DMEM supplemented with 15\% FBS, $1 \%$ nonessential amino acids, $1 \mathrm{mmol} / \mathrm{L}$ sodium pyruvate, 0.1 $\mathrm{mmol} / \mathrm{L} \mathrm{2-mercaptoethanol} \mathrm{and} 2 \mathrm{mmol} / \mathrm{L}$ glutamine). The cells were then counted and reseeded onto $0.1 \%$ gelatin-coated plates $\left(4 \times 10^{5}\right.$ ESCs/well). To determinie the effects of HSCEXs on HSC differentiation, the ESCs were treated with culture medium only (control) or $40 \mathrm{ng} / \mathrm{mL}$ of HSC-EXs ${ }^{\text {con }}$, HSC-EXs ${ }^{\mathrm{sc}}$ or HSC-EXs ${ }^{\text {miR126 }}$ with or without soluble Jagged1 $(100 \mathrm{ng} / \mathrm{mL}$; R\&D systems, Minneapolis, MN, USA) for a 10-d differentiation period. HSC-EXs and Jagged1 were added to the culture medium every three days ${ }^{[9]}$. At the end of the experiment, the cells were collected for flow cytometry, RT-PCR and Western blot analyses. The miR126 level in the ESCs was determined after $24 \mathrm{~h}$ co-culture and on d 10 after differentiation.

\section{Flow cytometry analysis of the generated HSCs}

After the 10-d differentiation period, the cells were washed with PBS, digested with $0.25 \%$ trypsin-0.02\% EDTA, centrifuged and resuspended with $100 \mu \mathrm{L}$ PBS. Then, each sample was incubated with PE-conjugated CD117, FITC-conjugated Sca1, or efluor 660-conjugated CD34 for $30 \mathrm{~min}$ in the dark. FITC-conjugated IgG, PE-conjugated IgG and efluor 660-conjugated IgG were used as isotype controls. After incubation, all samples were analyzed by flow cytometry (FACS canto flow cytometer) ${ }^{[26]}$, and 50000 events were collected for data analysis. A gate (P1) was set on the FSC-A vs SSC-A plot for the collected cell population. A second gate (P2) was set on the CD117-conjugated PE-A vs SSC-A for the analysis of $\mathrm{CD} 117^{+}$cells. HSCs were defined as $\mathrm{CD} 117^{+} \mathrm{CD} 34^{+} \mathrm{Sca} 1^{+}$cells. The percentage of HSCs was calculated as $\mathrm{CD} 117^{+} \mathrm{CD} 34^{+} \mathrm{Sca} 1^{+}$ cells/total cells $\times 100 \%$.

\section{Isolating HSCs from the differentiating ESCs}

To determine whether the generated HSCs were functional, we used magnetic activated cell sorting (MACS) to isolate the generated HSCs with anti-CD117 microbeads (Miltenyi Biotec) according to the manufacturer's instructions. In brief, after the 10-d differentiation period, ESCs from different groups were harvested and incubated with anti-CD117 microbeads antibody (10 $\mu \mathrm{L}$ anti-CD117 microbeads per $10^{7}$ total cells) in a $100 \mu \mathrm{L}$ reaction volume for $20 \mathrm{~min}$ in the refrigerator. Then, the $\mathrm{CD} 117^{+}$cells in each group were separated using a magnet separator (DynaMag-2 magnet; Life Technologies) as previously reported ${ }^{[27]}$. The isolated $\mathrm{CD} 117^{+}$cells were used for the colony-forming unit (CFU) assay described below.

\section{Hematopoietic colony-forming unit assay}

The colony-forming unit (CFU) assay is a commonly used in vitro functional assays for $\mathrm{HSCs}^{[28]}$. The $\mathrm{CFU}$ assay for the isolated $\mathrm{CD}_{117^{+}}$HSCs was performed using MethoCult ${ }^{\mathrm{TM}}$ medium (Stem Cell Technologies) according to the manufacture's instructions. Briefly, $1 \times 10^{4}$ dissociated single CD $117^{+}$ cells were mixed with methylcellulose medium supplemented with stem cell factor $(100 \mathrm{ng} / \mathrm{mL})$, granulocyte-macrophageCSF (100 ng/mL), IL-3 (10 ng/mL), IL-6 (10 ng/mL), granulocyte colony-stimulating factor $(100 \mathrm{ng} / \mathrm{mL})$, and erythropoietin (4 units $/ \mathrm{mL}$ ) in a $35 \mathrm{~mm}$ petri dish and cultured for $12 \mathrm{~d}$. The number of colonies formed was counted under an inverted microscope (EVOS, 10× magnification).

\section{Quantitative RT-PCR analysis}

Total RNA was extracted from cells using Tri-reagent (Sigma). The cDNA was generated by reverse transcription with a cDNA synthesis kit (Qiagen) in a $20 \mu \mathrm{L}$ volume according to manufacturer's instructions. The qRT-PCR analysis was performed by using SYBR green II qPCR Premix (Takara, Japan) in 96-well PCR plates on an ABI PRISM 7500 real-time PCR system (Bio-tek). The PCR conditions were $95^{\circ} \mathrm{C}$ for $10 \mathrm{~min}$ and 50 cycles at $95^{\circ} \mathrm{C}$ for $30 \mathrm{~s}, 60^{\circ} \mathrm{C}$ for $30 \mathrm{~s}$, and $72{ }^{\circ} \mathrm{C}$ for 1 min. U6 was used as the internal control. The relative expression of each gene was calculated using the $2^{-\Delta \Delta C \mathrm{~T}}$ method $^{[29]}$.

\section{Western blot analysis}

After the 10-d differentiation period, cell proteins were extracted with cell lysis buffer (Thermo Fisher Scientific) supplemented with a protease inhibitor tablet (Roche). Protein lysates were electrophoresed with SDS-PAGE and transferred onto PVDF membranes. The membranes were blocked with $5 \%$ non-fat milk for $1 \mathrm{~h}$ and incubated with primary antibodies against notch intracellular domain (NCID, 1:500; Abcam), Hes1 (1:100; Santa Cruz) or $\beta$-actin (1:4000; Sigma) at $4{ }^{\circ} \mathrm{C}$ overnight. After washing with T-PBS, the membranes were incubated with horseradish-peroxidase-conjugated IgG (Jackson Immuno Research Lab) for $1 \mathrm{~h}$ at RT. Blots were developed with enhanced chemiluminescence developing solutions and quantified using ImageJ software.

\section{Statistical analysis}

Each experimental measurement was repeated four times. The data are expressed as the mean \pm SEM. Multiple comparisons were analyzed by one- or two-way ANOVA followed by an LSD post-hoc test. SPSS 17.0 statistical software was used. For all measurements, $P<0.05$ was considered significant.

\section{Results \\ MiR126 mimics increased miR126 levels in HSCs and their released EXs}

After 24-h transfection with sc or miR126-mimics, HSCs were collected for real time RT-PCR analysis. As shown in Figure 1A, the miR126 level was significantly increased in HSCs transfected with miR126-mimics when compared to that in HSCs transfected with control or sc. These results suggested the successful miR126 transfection.

Using real time RT-PCR, we confirmed that the HSCEXs $^{\text {miR126 }}$ had a higher level of miR126 expression than HSC- 
A

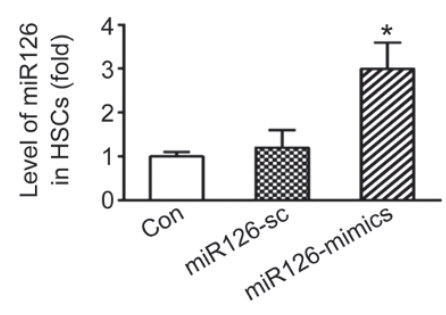

B

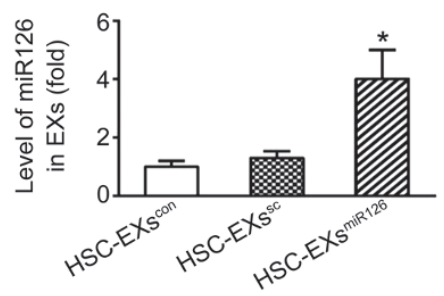

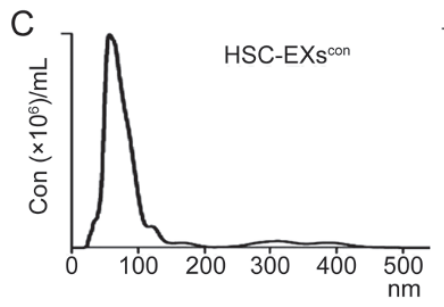
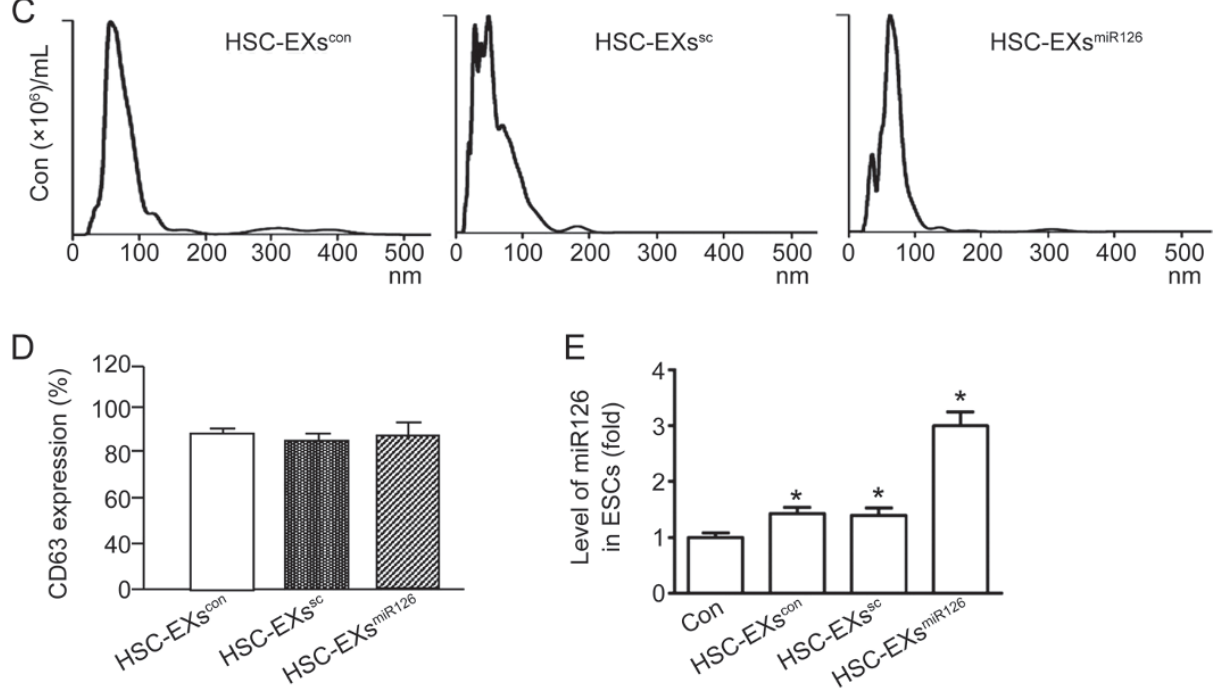

Figure 1. Analysis of miR126 expression in HSCs, HSC-EXs and ESCs co-cultured with various types of HSC-EXs. (A) miR126-mimic transfection increased the level of miR126 expression in HSCs. (B) HSC-EXs released from HSCs transfected with miR126 mimics carried a high level of miR126. (C) Representative image showing the size distribution of HSC-EXs analyzed by NTA. (D) CD63 expression in the HSC-EXs. (E) miR126 levels in the ESCs after $24 \mathrm{~h}$ co-culture. ${ }^{*} P<0.05$ vs con. Data are expressed as the mean \pm SEM. $n=4$.

EXs $^{\text {con }}$ and HSC-EXs ${ }^{\text {sc }}$ did (Figure 1B), which suggested successful enrichment of miR126 expression in the HSCEXs. Meanwhile, the sizes of the HSC-EXs collected from the culture medium were assessed by nanoparticle tracking analysis using the NS300 instrument. As shown in Figure $1 \mathrm{C}$, the sizes of the HSC-EXs ${ }^{\text {con }}$, HSC-EXs ${ }^{\text {miR126 }}$, and HSC-EXs ${ }^{\text {sc }}$ ranged from $30-120 \mathrm{~nm}$ in diameter, which was consistent with previous reports by others ${ }^{[7,9]}$ and $\mathrm{us}^{[25]}$. In addition, we characterized the HSC-EXs using the EX specific marker CD63. Our results showed that over $85 \%$ of EXs expressed CD63 (Figure 1D).

\section{HSC-EXs ${ }^{\text {miR126 }}$ co-culture increased miR126 expression in ESCs}

After 24-h co-culture with HSC-EXs ${ }^{\text {miR126 }}$, we determined the miR126 levels in ESCs. As shown in Figure 1E, the miR126 levels in ESCs were significantly elevated following HSC$\mathrm{EXS}^{\mathrm{con}}$ and HSC-EXs ${ }^{\mathrm{sc}}$ co-culture compared with the control. The expression of miR126 was further increased by HSC$\mathrm{EXS}^{\mathrm{miR} 126}$ co-culture.

HSC-EXs ${ }^{\text {miR126 }}$ promoted hematopoietic differentiation of ESCs via the miR126/Notch1 pathway to a greater extent than HSCEXs $^{\text {con }}$ and HSC-EXs ${ }^{\text {sc }}$ did

ESCs were induced for HSC differentiation in the presence of various HSC-EXs. The differentiation rate was analyzed by flow cytometry using the hematopoietic specific markers CD34, CD117 and Sca1. As shown in Figure 2, the percentage of HSCs (defined as $\mathrm{CD} 34^{+} \mathrm{CD} 117^{+} \mathrm{Sca} 1^{+}$cells) was higher in the HSC-EXs ${ }^{\text {con }}$ treatment group than in the control. We also found that HSC-EXs ${ }^{\text {miR126 }}$ treatment further remarkably increased ESC hematopoietic differentiation (versus HSC$\mathrm{EXS}^{\mathrm{sc}}$ ). The Notch1 ligand Jagged1 did not significantly change the ESC differentiation rate (versus control), although, it significantly abolished the effect of HSC-EXs ${ }^{\text {miR126 }}$ on promoting hematopoietic differentiation.

The generated HSCs had self-renewal capability

After the 10-d differentiation period, the generated HSCs were isolated by MACS with anti-CD117 microbeads to analyze their self-renewal ability. The CFU assay showed that the generated HSCs were able to form colony units after 12-d culture in methylcellulose medium (Figure 3).

HSC-EXs ${ }^{\text {miR126 }}$ modulated the expression of pluripotent/ hematopoietic and mesoderm commitment genes through the miR126/Notch1 pathway to a greater extent than HSC-EXs ${ }^{\text {con }}$ and HSC-EXs ${ }^{\text {sc }}$ did

We analyzed the expression of the pluripotency-related gene 
A
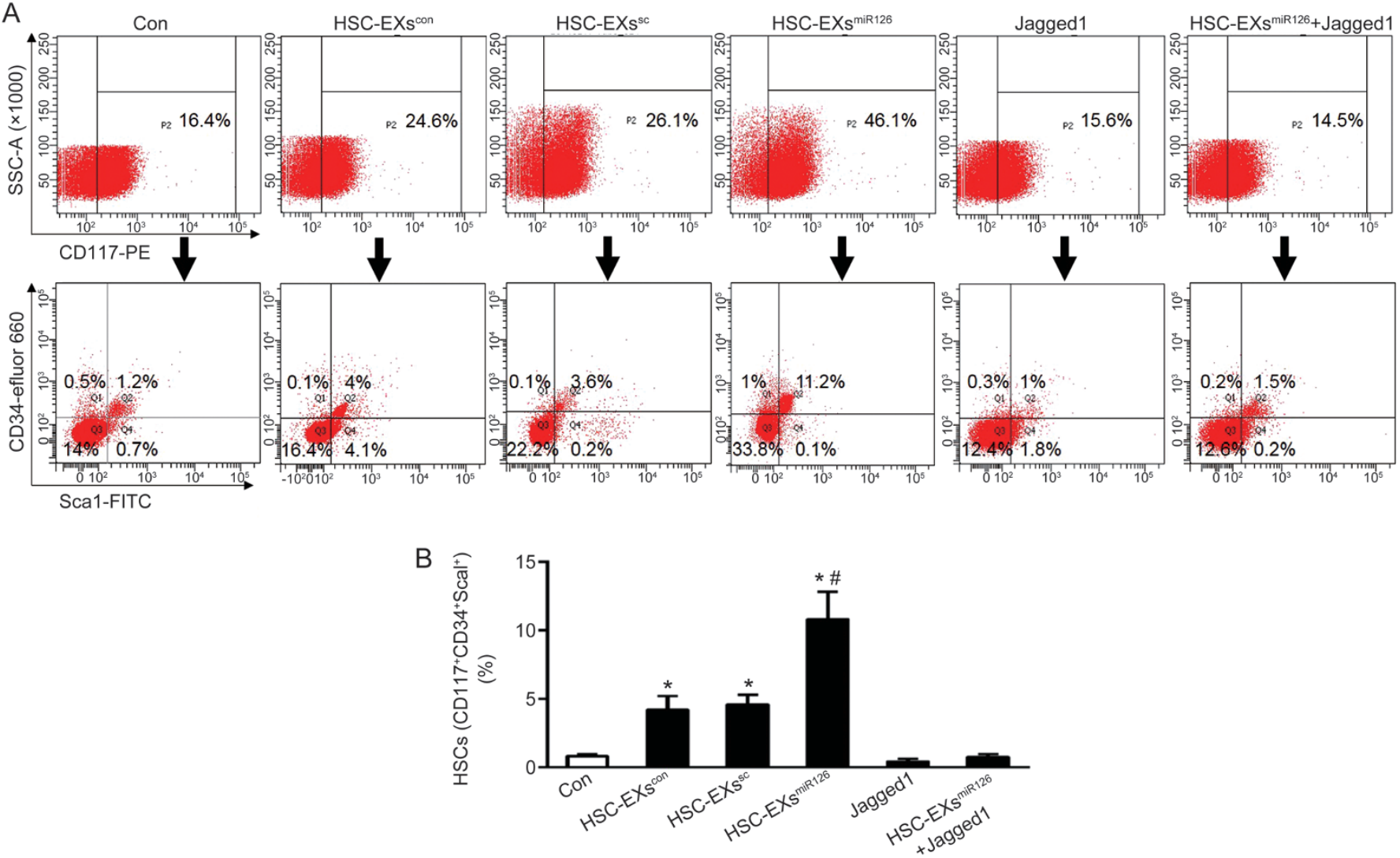

Figure 2. HSC-EXs ${ }^{\text {miR126 }}$ co-culture remarkably promoted the differentiation of ESCs into HSCs. (A) Representative flow cytometry plots showing the percentage of HSCs after 10-d differentiation. (B) Summarized data showing that HSC-EXs ${ }^{\text {con }}$ and HSC-EXs ${ }^{\text {sc }}$ treatment promoted the differentiation of ESCs into HSCs, with the greatest differentiation promoted by HSC-EX ${ }^{\text {miR126. }}$. This effect was attenuated by Jagged1. ${ }^{*} P<0.05$ vs Con. ${ }^{\#} P<0.05$ vs HSC$\mathrm{EXS}^{\mathrm{con}}$, or HSC-EXs${ }^{\mathrm{sc}}$, or Jagged1, or HSC-EXs ${ }^{\text {miR126}}+$ Jagged1. Data are expressed as the mean $\pm \mathrm{SEM} . n=4$.

SSEA1 and hematopoietic lineage-related genes CD117 and Sca1 in the differentiating ESCs. Our data (Figure 4A) showed that the mRNA level of SSEA1 was decreased, whereas the mRNA levels of CD117 and Sca1 were increased in the differentiating ESCs treated with HSC-EX ${ }_{s}^{\text {con }}$, HSC-EX ${ }^{\text {sc }}$ or HSC$\mathrm{EXS}^{\mathrm{miR} 126}$. The HSC-EXs ${ }^{\text {miR126 }}$ exhibited stronger effects than HSC-EXs ${ }^{\text {con }}$ or HSC-EXs ${ }^{\text {sc }}$ did. Activation of Notch1 pathway with Jagged1 alone did not change the mRNA levels of SSEA1, CD117 or Sca1 (versus control), whereas, it significantly abolished the effects of HSC-EXs ${ }^{\text {miR126 }}$ on the mRNA levels of SSEA1, CD117 and Sca1.

We also measured the expression of the germ-layer early commitment genes Nes (Nestin, for ectoderm), Bry (Brachyury, for mesoderm) and Afp (a-feto protein, for endoderm) in various HSC differentiation groups. We found that HSC$\mathrm{EXS}^{\mathrm{con}}$ or HSC-EXs ${ }^{\mathrm{sc}}$ treatment alone significantly increased the mRNA expression of Bry, which was further increased by HSC-EXs ${ }^{\text {miR126 }}$ treatment. Jagged1 blocked the increase in Bry mRNA expression elicited by HSC-EXs ${ }^{\text {miR126 }}$ and upregulated the mRNA levels of Nes in the differentiating ESCs. No significant changes in Afp expression were observed, suggesting that HSC-EXs could not affect endoderm differentiation of ESCs in our cell culture system (Figure 4B).
HSC-EXs ${ }^{\text {miR126 }}$ up-regulated miR126 expression and downregulated Notch1 mRNA levels in ESCs on d 10

As shown in Figure 5A, HSC-EXs ${ }^{\text {con }}$ and HSC-EXs ${ }^{\text {sc }}$ increased the miR126 levels in the differentiating ESCs. HSC-EXs ${ }^{\text {miR126 }}$ treatment remarkably increased the expression of miR126, which was not altered by Jagged1. These data suggested that HSC-EXs can modulate the expression of miR126 in differentiating ESCs.

To explore whether the Notch signaling pathway was involved in this process, we analyzed the mRNA expression levels of Notch receptors 1, 2 and 4 on the differentiating ESCs. We found that the HSC-EX ${ }^{\text {con }}$ and HSC-EX ${ }^{\text {sc }}{ }^{\text {signifi- }}$ cantly decreased the mRNA levels of Notch1. HSC-EXs ${ }^{\text {miR126 }}$ decreased Notch1 levels to the greatest extent, and this effect was blocked by Jagged1. The expression levels of Notch2 and Notch4 were not different among groups (Figure 5B). These findings suggested that HSC-EXs ${ }^{\text {miR126 }}$ promoted HSC differentiation by inhibiting the Notch1 pathway.

Enrichment of miR126 in HSC-EXs promoted the downregulation of NCID/Hes1 in ESCs on d 10

As shown in Figure 6A, NCID expression was significantly decreased in the differentiating ESCs treated with HSC- 

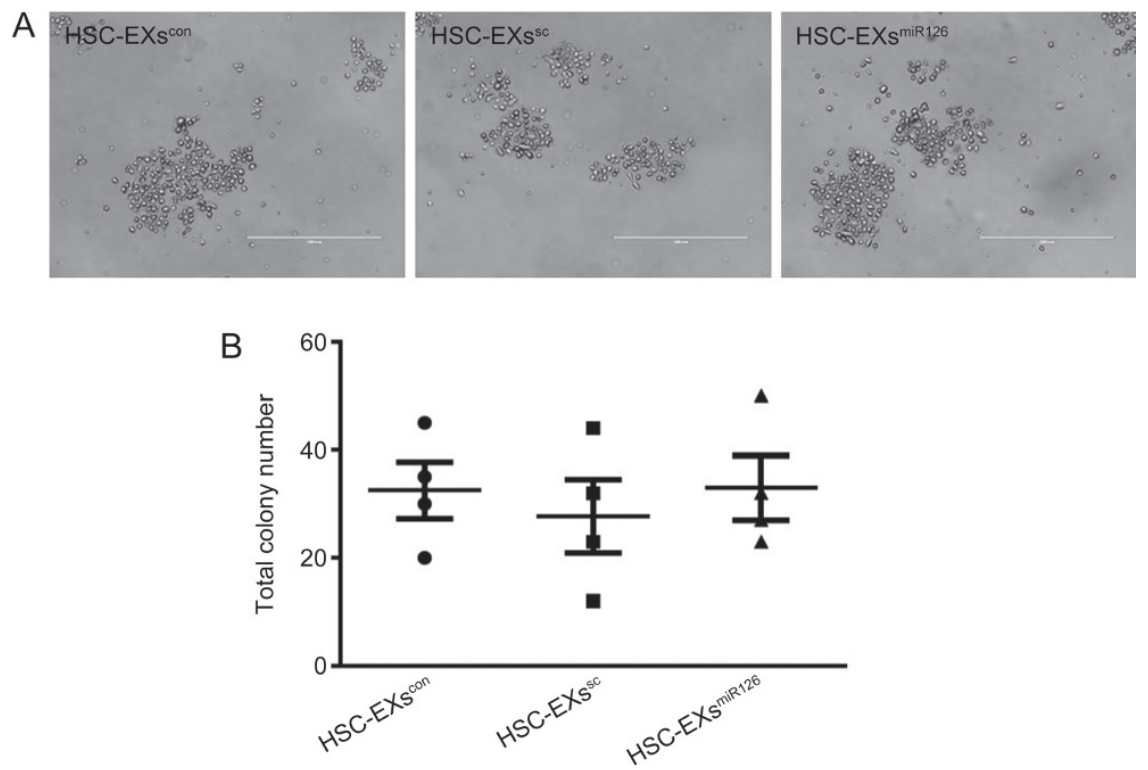

Figure 3. Isolated $\mathrm{CD} 117^{+}$cells had colony-forming abilities. (A) Representative images showing the colonies in different groups. (B) Summarized data showing the total number of colonies produced by the isolated $\mathrm{CD} 117^{+}$cells on methylcellulose medium. Each square represents the total colonies from one plate.

EXs ${ }^{\text {con }}$ or HSC-EXss ${ }^{\text {sc }}$ as compared with that of the control group in, which NCID expression was further downregulated by HSC-EXs ${ }^{\text {miR126. }}$. Jagged1 alone increased NCID expression and reversed the downregulation of NCID induced by HSC-EXs ${ }^{\text {miR126 }}$. These data suggest that HSC-EXs ${ }^{\text {miR126 }}$ can inhibit Notch signaling in the differentiating ESCs.

Hes1 is a major downstream gene of the Notch1 signaling pathway. Compare with the HSC-EXs ${ }^{\mathrm{con}}$ and HSC-EXs ${ }^{\mathrm{sc}}$, HSC-EXs ${ }^{\text {miR126 }}$ decreased Hes1 mRNA to a greater extent in the differentiating cells. Jagged 1 inhibited this effect ( $v S$ HSC-EXs $\left.{ }^{\text {miR126 }}, P<0.05\right)$. Similarly, Western blot analysis demonstrated that the expression of Hes1 was down-regulated by HSC-EXs ${ }^{\text {con }}$ and HSC-EXs ${ }^{\text {sc }}$. The HSC-EXs ${ }^{\text {miR126 }}$ further decreased Hes1 expression in the differentiating ESCs, and this effect was blocked by Jagged1 (Figure 6B, 6C). The data further confirmed that HSC-EXs can modulate the miR126/
Notch/Hes1 pathway.

\section{Discussion}

The major findings of the present study are that HSC-EXs can promote HSC differentiation from ESCs and that overexpression of miR126 can increase the efficiency of HSC-EXsmediated differentiation. In addition, our data suggested that miR126-mediated inhibition of the Notch1/Hes1 pathway is the underlying mechanism for this process.

EXs, which are small vesicles derived from intracellular multivesicular bodies of various cell types ${ }^{[3,30]}$, are enriched in bioactive molecules such as proteins, mRNAs and miRs from the cells of origin. EXs are released constitutively or in response to different stimuli and impact the genetic content and function of recipient cells by delivering bioactive molecules $^{[4,6,7]}$. In this study, we found that HSC-EXs co-culture
A

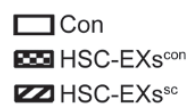

HSC-EXs ${ }^{\text {miR126 }}$

Jagged 1

HSC-EXs ${ }^{\text {miR126}+J a g g e d 1 ~}$

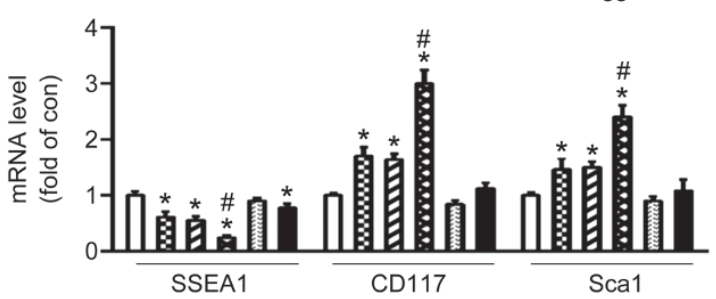

B

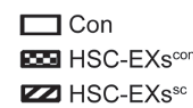

HSC-EXs ${ }^{\text {miR } 126}$

Jagged 1

HSC-EXs ${ }^{\text {miR } 126}+$ Jagged 1

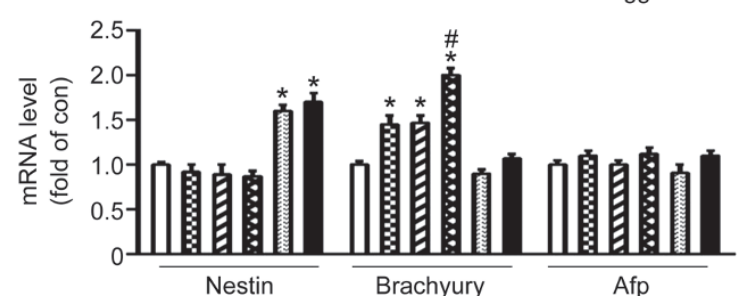

Figure 4. RT-PCR analysis of specific lineage-related genes from ESCs after 10-d differentiation. (A) The mRNA level of the pluripotent gene SSEA1 was decreased, whereas the mRNA levels of the HSC genes CD117 and Sca1 were increased in the ESCs treated with HSC-EXS ${ }^{\text {con }}$ and HSC-EXs ${ }^{\text {sc }}$ that underwent 10-d differentiation. HSC-EXs ${ }^{\text {miR126 }}$ enhanced HSC differentiation which was abolished by Jagged1. (B) The mRNA levels of markers in three germ layers, Nes (ectoderm), Bry (mesoderm) and Afp (endoderm). HSC-EX ${ }^{\text {miR126 }}$ increased mRNA level of Bry, which was inhibited by Jagged1. * $P<0.05$ vs Con. ${ }^{\#} P<0.05$ vs HSC-EXs ${ }^{\text {con }}$, or HSC-EXs ${ }^{\text {sc }}$, or Jagged1, or HSC-EXs ${ }^{\text {miR126}}+$ Jagged1. Data are expressed as the mean \pm SEM. $n=4$. 

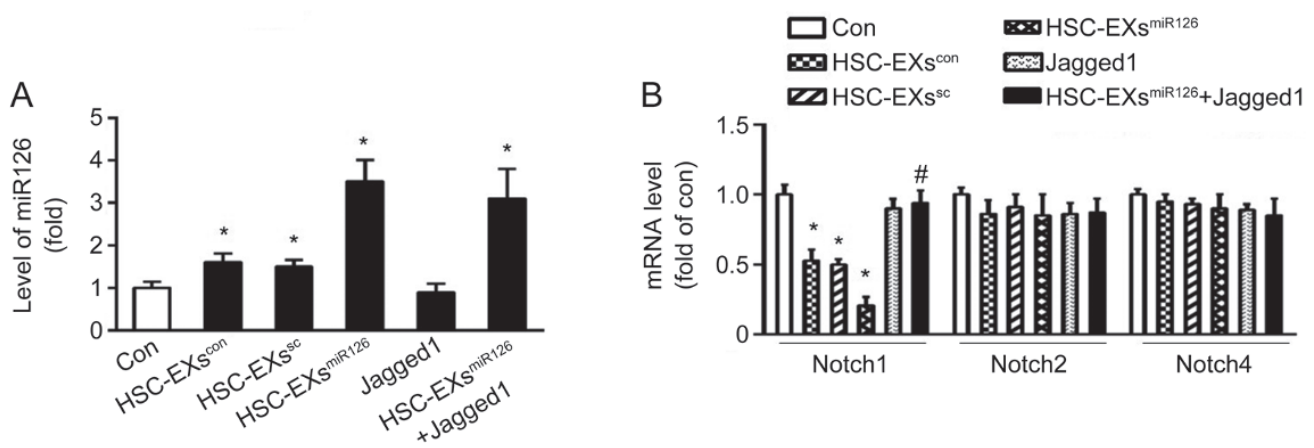

Figure 5. HSC-EXs increased miR126 levels and decreased mRNA levels of Notch1 in ESCs on d 10. (A) HSC-EXs increased the level of miR126 in the differentiating ESCs. (B) The summarized data showing that the expressions of Notch1, Notch2 and Notch4 in the cells that underwent 10-d differentiation. ${ }^{*} P<0.05$ vs Con. ${ }^{\#} P<0.05$ vs HSC-EXs ${ }^{\text {con }}$, HSC-EXs ${ }^{\text {sc }}$, HSC-EXs ${ }^{\text {miR126, }}$ or Jagged1. Data are expressed as the mean \pm SEM. $n=4$.

promoted the differentiation of ESCs into $\mathrm{CD} 117^{+} \mathrm{CD} 34^{+} \mathrm{Sca} 1^{+}$ HSCs, indicating the important role of EXs in hematopoietic differentiation. This finding is supported by previous studies that showed the participation of EXs in neuronal ${ }^{[9]}$ or macrophage differentiation ${ }^{[10]}$, which represents a novel feedback mechanism from the differentiated cells.

It is well known that miRs are important regulators of hematopoiesis, and miR126 is highly expressed in HSC compartments and downregulated during hematopoietic commitment ${ }^{[14]}$. Our previous study demonstrated that EX-carried miR126 can be delivered to endothelial cells ${ }^{[4]}$. Nevertheless, whether miR126 participates in the hematopoietic differentia- tion of ESCs elicited by HSC-EXs remains elusive. We determined that miR126 expression was higher in the HSC-EXs ${ }^{\text {con }}$ co-cultured cells. To further explore the role of miR126 in the differentiation of ESCs in HSCs, we overexpressed miR126 in HSCs to obtain EXs with high levels of miR126 (HSC$\left.\mathrm{EXs}^{\mathrm{miR} 126}\right)$. Interestingly, our data revealed that HSC-EXs ${ }^{\text {miR126 }}$ remarkably increased the ESC hematopoietic differentiation rate, indicating that miR126 is the major underlying mechanism responsible for the effect of HSC-EXs on ESC differentiation. However, whether miR126 alone can affect hematopoietic differentiation of ESCs requires further study. Meanwhile, knock down of miR126 using inhibitors could be used

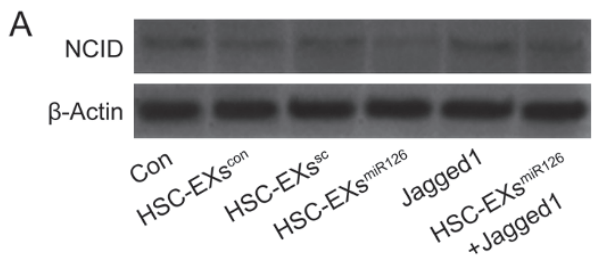

$\mathrm{B}$
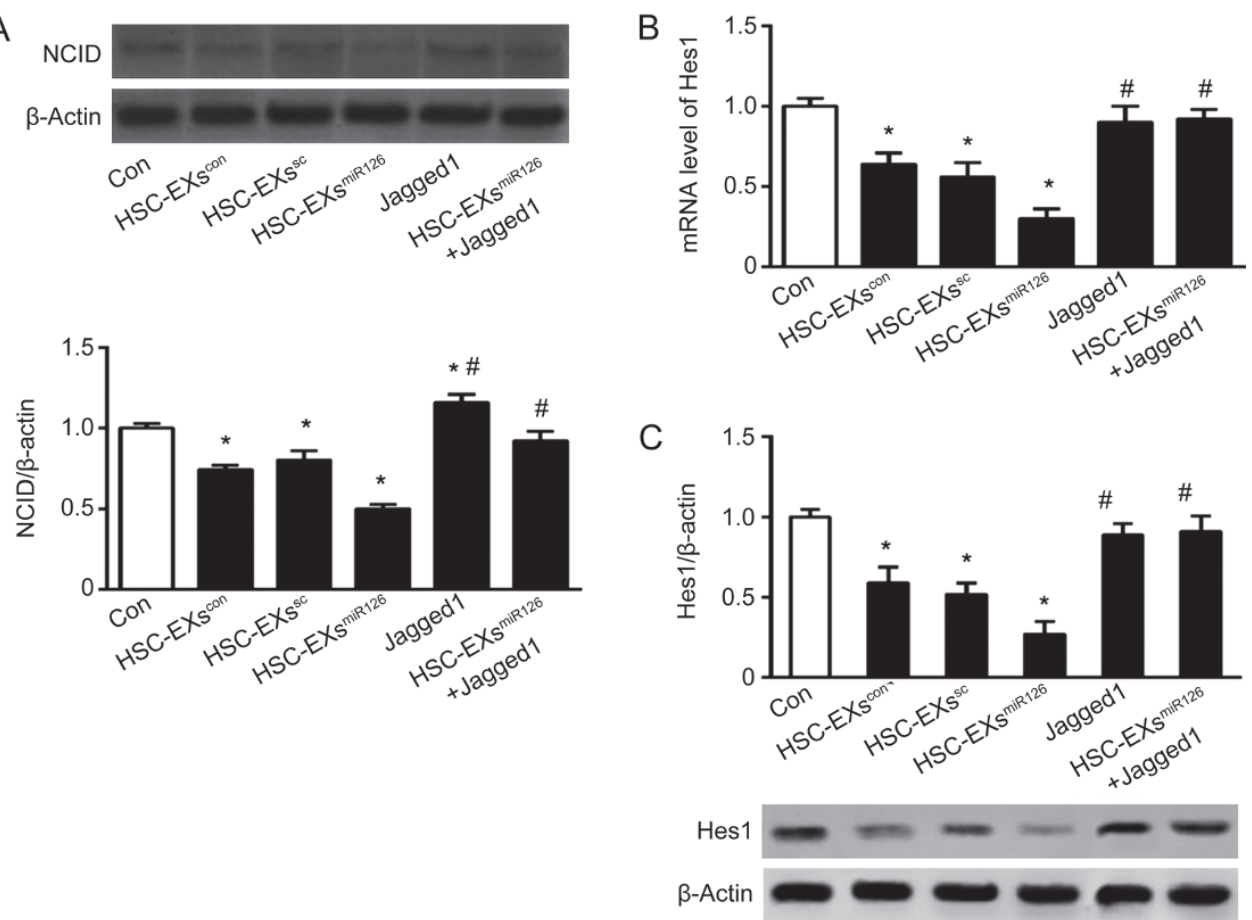

Figure 6. HSC-EXs down-regulated NCID expression and the mRNA and protein levels of Hes1 in ESCs on d 10. (A) Top: representative bands of NCID and $\beta$-actin. Bottom: summarized data showing the expression of NCID in the differentiating cells; $(B, C)$ The summarized data showing the mRNA and protein levels of Hes1 in the differentiating ESCs. ${ }^{*} P<0.05$ vs Con. ${ }^{\#} P<0.05$ vs HSC-EXs ${ }^{\text {con }}$, HSC-EXs ${ }^{\text {sc }}$, or HSC-EXs ${ }^{\text {miR126 }}$. Data are expressed as the mean \pm SEM. $n=4$. 
to further confirm the role of miR126 in ESC hematopoietic differentiation. Whether other sources of EXs such as endothelial cell-derived EXs and fibroblast cell derived EXs could offer such a regulatory effect requires more investigation. In the present study, to test whether the generated HSCs were functional, we isolated the generated HSCs using MACS with anti-CD117 conjugated microbeads and performed a colony formation unit (CFU) assay in vitro. The generated HSCs were capable of forming colony units on methylcellulose medium. Meanwhile, we found that there was no difference in colony unit formation among the HSCs generated from ESCs treated with HSC-EXs ${ }^{\text {con }}$, HSC-EXs ${ }^{\mathrm{sc}}$, or HSC-EXs ${ }^{\text {miR126}}$. These findings suggested that miR126 can promote HSC differentiation from ESCs but does not affect the function of the generated HSCs.

Previous studies have revealed that activation of Notch signaling in ESCs leads to preferential neuroectoderm fate determination, whereas inactivation of this pathway facilitates the mesodermal fate ${ }^{[31-33]}$. In this study, we found that ESCs cocultured with HSC-EXs ${ }^{\text {con }}$ or HSC-EXs $s^{\text {sc }}$ promoted differentiation into the Brachyury-positive mesodermal lineage, and the HSC-EXs ${ }^{\text {miR126 }}$ enhanced this effect. These data are line with the observations made using the hematopoietic specific markers CD117 and Sca1. Here, we found that Jagged1 blocked the effect of HSC-EXs ${ }^{\text {miR126 }}$ and promoted the expression of Nestin (ectoderm lineage) in the differentiating ESCs, which is in agreement with a previous study showing that overexpression of Jagged1 can promote the differentiation of ESCs under neural differentiation induction ${ }^{[18]}$. Collectively, these data indicated that HSC-EXs can regulate fate choice in ESCs through the miR126/Notch signaling pathway.

Cellular receptor expression varies among different cell types, which elicits distinct responses during differentiation process. Jagged1-mediated Notch signaling results in enhanced Hes5 expression and diminished Hey1 expression, leading to the differentiation of ESCs toward a neural lineage, whereas, Delta-like 4 reverses the expression pattern of these genes and results in mesodermal commitment of ESCs ${ }^{[18]}$. In this study, we found that HSC-EXs ${ }^{\text {miR126 }}$ co-culture downregulated Notch1 mRNA levels, which led to the differentiation of ESCs towards HSCs. Our findings are supported by a previous study indicating that ectopic expression of Notch intracellular domain suppressed hematopoietic differentiation of $\mathrm{FLK}^{+}$mesodermal progenitors ${ }^{[34]}$.

It is well-known that the Notch signaling involves the Hes and Hey family of transcription co-repressors downstream. Hes1 is a direct target gene of Notch and is highly expressed in ESCs ${ }^{[35,36]}$. Our results showed that expression of Hes1 in the differentiating cells was significantly decreased by HSC$\mathrm{EXs}^{\mathrm{con}}$ and HSC-EXs ${ }^{\mathrm{sc}}$ and more so by HSC-EXs ${ }^{\mathrm{miR} 126}$. These findings suggested that enrichment of miR126 can enhance the inhibition of HSC-EXs on Notch signaling in the differentiating ESCs. To directly elucidate the relationship between HSC-EXs ${ }^{\text {miR126 }}$ and the Notch pathway, we added Jagged1, a Notch ligand, to activate the Notch pathway in the differentiating ESCs. As expected, Jagged1 activated Notch signal as evidenced by the increased level of NCID expression. More- over, it reversed the downregulation of Hes1 induced by HSC$\mathrm{EXs}^{\mathrm{miR} 126}$, which supported the observation that HSC-EXs ${ }^{\text {miR126 }}$ can inhibit the Notch pathway. Taken together, these findings suggest that HSC-EXs can promote HSC differentiation through the miR126-modulated Notch1/Hes1 pathway.

\section{Conclusions}

In conclusion, HSC-EXs accelerate HSC differentiation from ESCs by modulating the miR126/Notch1 signal pathway, providing a novel approach to increase the production of HSCs for clinical use.

\section{Acknowledgements}

This work was supported by National Natural Science Foundation of China (№ 81400360); the Science and Technology Planning Project of Zhanjiang (№ [2012] 172); and the Competitive Project of Zhanjiang (2014A01022).

\section{Author contribution}

Yanfang CHEN, Bin ZHAO, Ji BIHL, Yi YANG and Riling CHEN designed the experiments; Feng-ling LIAO, Lin TAN and Xiao-tang MA performed the experiments; Fengling LIAO, Lin TAN, Xiao-tang MA, Ri-ling CHEN, Jin-ju WANG, Yanfang CHEN and Ji BIHL analyzed the data; Fengling LIAO, Lin TAN, Hua LIU, Jin-ju WANG, Bin ZHAO, Yi YANG, Ri-ling CHEN and Yanfang CHEN wrote the paper.

\section{References}

1 Gori JL, Butler JM, Chan YY, Chandrasekaran D, Poulos MG, Ginsberg $\mathrm{M}$, et al. Vascular niche promotes hematopoietic multipotent progenitor formation from pluripotent stem cells. J Clin Invest 2015; 125: 1243-54.

2 McKinney-Freeman S, Daley G. Derivation of hematopoietic stem cells from murine embryonic stem cells. J Vis Exp 2007; (2): 162.

3 Katsuda T, Kosaka N, Takeshita F, Ochiya T. The therapeutic potential of mesenchymal stem cell-derived extracellular vesicles. Proteomics 2013; 13: 1637-53.

4 Wang J, Chen S, Ma X, Cheng C, Xiao X, Chen J, et al. Effects of endothelial progenitor cell-derived microvesicles on hypoxia/ reoxygenation-induced endothelial dysfunction and apoptosis. Oxid Med Cell Longev 2013; 2013: 572729.

5 Emanueli C, Shearn AI, Angelini GD, Sahoo S. Exosomes and exosomal miRNAs in cardiovascular protection and repair. Vascul Pharmacol 2015; 71: 24-30.

6 Mause SF, Weber C. Microparticles: protagonists of a novel communication network for intercellular information exchange. Circ Res 2010; 107: 1047-57.

7 Xin H, Li Y, Liu Z, Wang X, Shang X, Cui Y, et al. MiR-133b promotes neural plasticity and functional recovery after treatment of stroke with multipotent mesenchymal stromal cells in rats via transfer of exosome-enriched extracellular particles. Stem Cells 2013; 31: 2737-46.

8 Wu K, Yang Y, Zhong Y, Ammar HM, Zhang P, Guo R, et al. The effects of microvesicles on endothelial progenitor cells are compromised in type 2 diabetic patients via downregulation of the miR-126/VEGFR2 pathway. Am J Physiol Endocrinol Metab 2016; 310: E828-37.

9 Takeda YS, Xu Q. Neuronal differentiation of human mesenchymal stem cells using exosomes derived from differentiating neuronal cells. 
PLoS One 2015; 10: e0135111.

10 Ismail N, Wang Y, Dakhlallah D, Moldovan L, Agarwal K, Batte K, et al. Macrophage microvesicles induce macrophage differentiation and miR-223 transfer. Blood 2013; 121: 984-95.

11 Huang X, Gschweng E, Van HB, Cheng D, Mikkola HK, Witte ON. Regulated expression of microRNAs-126/126* inhibits erythropoiesis from human embryonic stem cells. Blood 2011; 117: 2157-65.

12 Chen CZ, Li L, Lodish HF, Bartel DP. MicroRNAs modulate hematopoietic lineage differentiation. Science 2004; 303: 83-6.

13 Nikolic I, Plate KH, Schmidt MH. EGFL7 meets miRNA-126: an angiogenesis alliance. J Angiogenes Res 2010; 2: 9.

14 Lechman ER, Gentner B, van Galen P, Giustacchini A, Saini M, Boccalatte FE, et al. Attenuation of miR-126 activity expands HSC in vivo without exhaustion. Cell Stem Cell 2012; 11: 799-811.

15 Huang F, Zhu X, Hu XQ, Fang ZF, Tang L, Lu XL, et al. Mesenchymal stem cells modified with miR-126 release angiogenic factors and activate Notch ligand Delta-like-4, enhancing ischemic angiogenesis and cell survival. Int J Mol Med 2013; 31: 484-92.

16 Dikic I, Schmidt MH. Notch: Implications of endogenous inhibitors for therapy. Bioessays 2010; 32: 481-7.

17 Bray SJ. Notch signalling: a simple pathway becomes complex. Nat Rev Mol Cell Biol 2006; 7: 678-89.

18 Ramasamy SK, Lenka N. Notch exhibits ligand bias and maneuvers stage-specific steering of neural differentiation in embryonic stem cells. Mol Cell Biol 2010; 30: 1946-57.

19 Pui JC, Allman D, Xu L, DeRocco S, Karnell FG, Bakkour S, et al. Notch1 expression in early lymphopoiesis influences B versus $T$ lineage determination. Immunity 1999; 11: 299-308.

20 Li H, Yu B, Zhang Y, Pan Z, Xu W, Li H. Jagged1 protein enhances the differentiation of mesenchymal stem cells into cardiomyocytes. Biochem Biophys Res Commun 2006; 341: 320-5.

21 Stier S, Cheng T, Dombkowski D, Carlesso N, Scadden DT. Notch1 activation increases hematopoietic stem cell self-renewal in vivo and favors lymphoid over myeloid lineage outcome. Blood 2002; 99 : 2369-78.

22 Ohishi K, Varnum-Finney B, Bernstein ID. Delta-1 enhances marrow and thymus repopulating ability of human $\mathrm{CD} 34^{+} \mathrm{CD} 38$ cord blood cells. J Clin Invest 2002; 110: 1165-74.

23 Han W, Ye Q, Moore MA. A soluble form of human Delta-like-1 inhibits differentiation of hematopoietic progenitor cells. Blood 2000; 95 : 1616-25.

24 Murry CE, Keller G. Differentiation of embryonic stem cells to clinically relevant populations: lessons from embryonic development. Cell
2008; 132: 661-80.

25 Wang J, Guo R, Yang Y, Jacobs B, Chen S, Iwuchukwu I, et al. The novel methods for analysis of exosomes released from endothelial cells and endothelial progenitor cells. Stem Cells Int 2016; 2016: 2639728.

26 Challen GA, Boles N, Lin KK, Goodell MA. Mouse hematopoietic stem cell identification and analysis. Cytometry A 2009; 75: 14-24.

27 Wang J, Zhong Y, Ma X, Xiao X, Cheng C, Chen Y, et al. Analyses of endothelial cells and endothelial progenitor cells released microvesicles by using microbead and Q-dot based nanoparticle tracking analysis. Sci Rep 2016; 6: 24679.

28 Vodyanik MA, Thomson JA, Slukvin II. Leukosialin (CD43) defines hematopoietic progenitors in human embryonic stem cell differentiation cultures. Blood 2006; 108: 2095-105.

29 Pan Q, Liu H, Zheng C, Zhao Y, Liao X, Wang Y, et al. Microvesicles derived from inflammation-challenged endothelial cells modulate vascular smooth muscle cell functions. Front Physiol 2016; 7: 692.

30 Al-Nedawi K, Meehan B, Micallef J, Lhotak V, May L, Guha A, et al. Intercellular transfer of the oncogenic receptor EGFRvIII by microvesicles derived from tumour cells. Nat Cell Biol 2008; 10 : 619-24.

31 Lowell S, Benchoua A, Heavey B, Smith AG. Notch promotes neural lineage entry by pluripotent embryonic stem cells. PLoS Biol 2006; 4: e121.

32 Schroeder T, Fraser ST, Ogawa M, Nishikawa S, Oka C, Bornkamm GW, et al. Recombination signal sequence-binding protein Jkappa alters mesodermal cell fate decisions by suppressing cardiomyogenesis. Proc Natl Acad Sci U S A 2003: 100: 4018-23.

33 Nemir M, Croquelois A, Pedrazzini T, Radtke F. Induction of cardiogenesis in embryonic stem cells via downregulation of Notch1 signaling. Circ Res 2006; 98: 1471-8.

34 Huang C, Jackson M, Samuel K, Taylor AH, Lowell S, Forrester LM. Haematopoietic differentiation is inhibited when Notch activity is enhanced in FLK1 $1^{+}$mesoderm progenitors. Stem Cell Res 2013; 11 : 1273-87.

35 Jarriault S, Brou C, Logeat F, Schroeter EH, Kopan R, Israel A. Signalling downstream of activated mammalian Notch. Nature 1995; 377: 355-8.

36 Kobayashi T, Mizuno H, Imayoshi I, Furusawa C, Shirahige K, Kageyama R. The cyclic gene Hes1 contributes to diverse differentiation responses of embryonic stem cells. Genes Dev 2009; 23: $1870-5$. 